\title{
Evaluation by Sharp Indentation of Anisotropic Plastic Behaviour in Progressively Drawn Pearlitic Steel
}

\author{
Jesús TORIBIO, ${ }^{1)}$ Beatriz GONZÁLEZ, ${ }^{1)}$ Juan-Carlos MATOS ${ }^{21}$ and Viktor KHARIN ${ }^{11}$ \\ 1) Department of Materials Engineering University of Salamanca, 49022 Zamora, Spain. \\ 2) Department of Computing Engineering University of Salamanca, 49022 Zamora, Spain.
}

(Received on December 10, 2010; accepted on February 25, 2011)

\begin{abstract}
This paper analyzes the evolution of Vickers micro-hardness in progressively drawn eutectoid steels focussing the study on the characteristic sections of the wires. The average value of the Vickers microhardness was calculated for each section according to its symmetry, thus studying the variation of these values with cold drawing. The indentation marks after the tests demonstrate the anisotropy caused by the cold drawing process itself on the eutectoid steel, and a finite element modelling allows one to finish material characterization.
\end{abstract}

KEY WORDS: Vickers indentation; prestressing steel; plastic microdamage; plastic anisotropy.

\section{Introduction}

Hardness tests, traditionally used to evaluate material resistance to localised plastic strain, have many other applications, and may be used at different scales: macro, micro and nano. Materials characterization from hardness tests (multiaxial states) allows one to obtain the elastic-plastic parameters related to material behaviour in the tension test. From the hardness values, a prediction of the stress-strain curve is possible, ${ }^{1)}$ sometimes in the form of an equation similar to that proposed by Tabor $^{2)}$ with the existence of one or two deformations characteristic of the material. ${ }^{3)}$

In the instrumented tests, a plot of load versus penetration depth is obtained, thus allowing one to determine several parameters of the material characteristic curve. ${ }^{4,5)}$ The slope of the initial part of the unloading curve is related to the elastic properties of the material and the loading curve exhibits a parabolic dependence with the penetration depth of the imprint (Kick's law), whose relationship not purely quadratic is related to the size effect in the yielding phenomenon. ${ }^{6}$

From the curve load versus penetrator depth, two fitting procedures are possible in the matter of isotropic mechanical properties. To solve this uniqueness problem a study is necessary of the residual strain in the imprint boundary, cf., ${ }^{5)}$ the relationship being unique in some cases. ${ }^{7,8)}$ The importance of using two or more indentators has been demonstrated, apart from the error reduction in the calculations when the indentator angle diminishes. ${ }^{9}$ )

A correlation also exists between material properties and plastic strain in the imprint and its neighbourhoods after the hardness test. The phenomena of piling-up and sinking-in are related to the strain hardening coefficient. ${ }^{3,10)}$ Anisotropic materials require the analysis of residual displacement as a consequence of their constitutive anisotropy. ${ }^{11)}$
In addition, hardness tests have been used to obtain the fields of residual stresses and strains in the material. ${ }^{12,13)}$ Residual stresses exhibit a little effect on the hardness but, on the other hand, the plastic strain fields exert a great influence on the hardness.

In this paper an analysis is presented of the evolution of Vickers microhardness in eutectoid steels coming from different stages of a real cold drawing process, analysing hardness and plastic damage in the two characteristic sections of the wire (transverse and longitudinal). A three dimensional (3D) modelling of the Vickers hardness test was designed by using the finite element method.

\section{Experimental Procedure}

Material used was progressively-drawn eutectoid pearlitic steel (Table 1). It was studied in its initial state (hot rolled material, not cold drawn at all), six intermediate stages and the final commercial product (prestressing steel, heavily cold drawn).

The nomenclature used to characterise the steels was a letter representing the chemical composition (B) and a digit indicating the number of drawing steps which has undergone. The steel was supplied in wire form and the degree of cold drawing is characterised by means of the cumulative plastic strain $\varepsilon^{\mathrm{P}}$ as a function of the diameter reduction according to the following expression,

$$
\varepsilon^{P}=2 \ln \frac{D_{0}}{D_{i}}
$$

where $D_{\mathrm{i}}$ is the diameter of the wire after $i$ drawing steps and $D_{0}$ that corresponding to the initial steel before cold drawing. The diameter $D$ of the wire decreases with the number of drawing steps, so that the cumulative plastic strain $\varepsilon^{\mathrm{P}}$ increases with the cold drawing degree (Table 2). 
Table 1. Chemical composition of steel B.

\begin{tabular}{cccccc}
\hline$\% \mathrm{C}$ & $\% \mathrm{Mn}$ & $\% \mathrm{Si}$ & $\% \mathrm{P}$ & $\% \mathrm{Cr}$ & $\% \mathrm{~V}$ \\
\hline 0.789 & 0.698 & 0.226 & 0.011 & 0.271 & 0.078 \\
\hline
\end{tabular}

Table 2. Diameter $(D)$ and cumulative plastic strain $\left(\varepsilon^{\mathrm{P}}\right)$.

\begin{tabular}{crrrrrrrr}
\hline Steel & \multicolumn{1}{c}{ B0 } & \multicolumn{1}{c}{ B1 } & \multicolumn{1}{c}{ B2 } & B3 & B4 & B5 & B6 & B7 \\
\hline$D(\mathrm{~mm})$ & 12.10 & 11.23 & 10.45 & 9.68 & 9.02 & 8.54 & 8.18 & 7.00 \\
$\varepsilon^{\mathrm{P}}$ & 0.00 & 0.15 & 0.29 & 0.45 & 0.59 & 0.70 & 0.78 & 1.10 \\
\hline
\end{tabular}

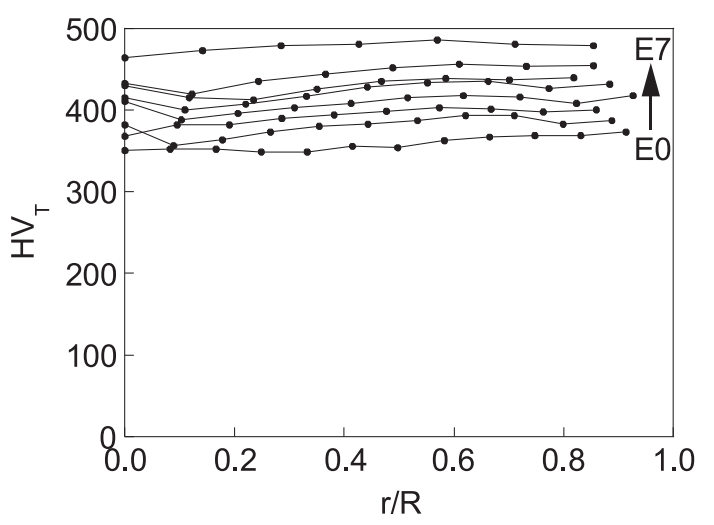

Fig. 1. Vickers hardness: transverse section.

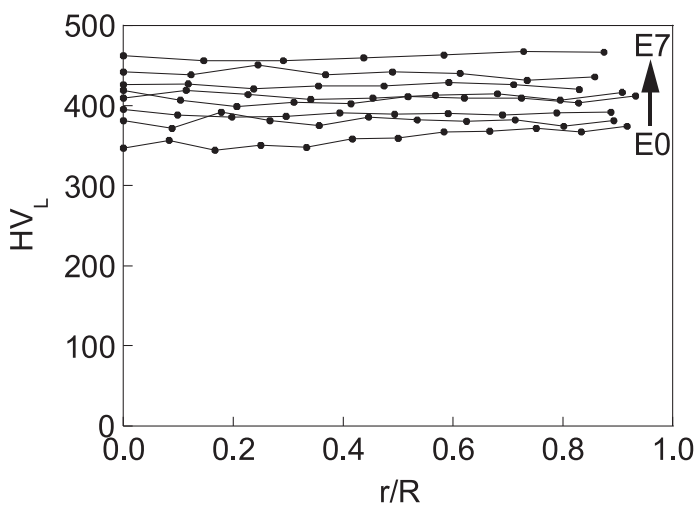

Fig. 2. Vickers hardness: longitudinal section.

Vickers test allows one to determine the existence of hardness variations in both the transverse $\left(S_{\mathrm{T}}\right)$ and the longitudinal $\left(S_{\mathrm{L}}\right)$ sections of the wires coming from different drawing stages. Microhardness was evaluated at six radial distances in both sections, applying a load of $19.714 \mathrm{~N}$ during 15 seconds, and maintaining a distance of $0.5 \mathrm{~mm}$ between measurements and enough separation from the specimen surface. The surfaces of analysis were grounded and polished to eliminate any impurity altering the results of the test.

\section{Experimental Results}

After the tests, the average values of the measurements through the dimensionless radius were represented, so that $r / R=0$ is associated with the specimen centre and $r / R=1$ with the sample surface. The hardness in the transverse $\left(H V_{\mathrm{T}}\right)$ and the longitudinal $\left(H V_{\mathrm{L}}\right)$ sections increases with the number of drawing steps (Figs. 1 and 2), with variations in relation to a reference horizontal straight line (associated with

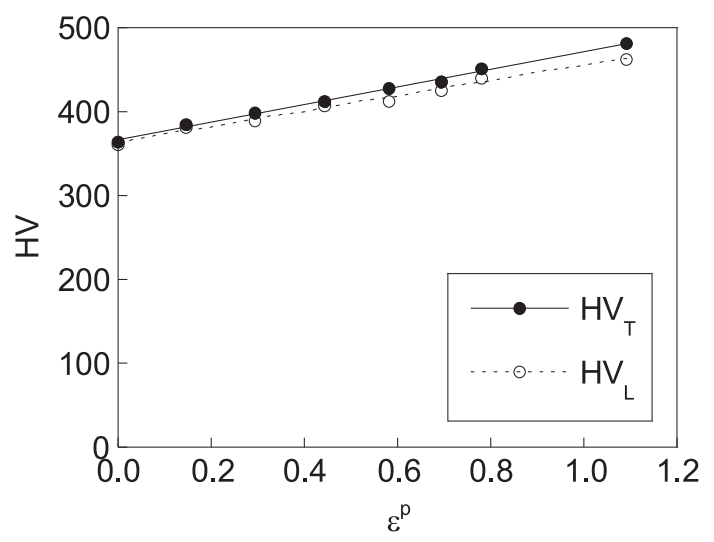

Fig. 3. Average Vickers hardness.

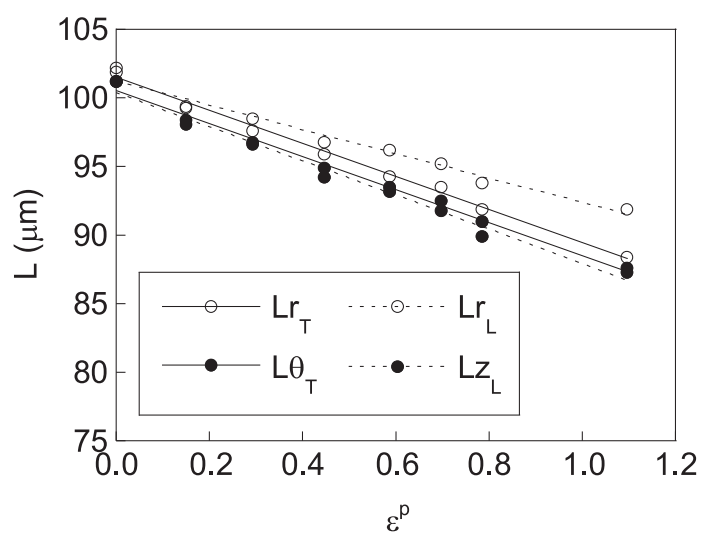

Fig. 4. Average diagonals.

a homogeneous material) which can be associated with the existence of elevated residual stresses as a consequence of the drawing process. ${ }^{14)}$ In the hot rolled bar and the prestressing steel wire such residual stresses are lower, in the first case because it is a rolled material (not cold drawn at all) and in the second case (final product) because it suffered a stress-relieving treatment.

The hardness and the imprint diagonals representative of each steel were calculated in both sections, taking into account the axisymmetry existent during the drawing process. When representing together the longitudinal and transverse Vickers hardness in the steels (Fig. 3), it is observed how both increase with the cold drawing. The hardness is really similar in the first stages of cold drawing in both sections, becoming a little bit greater in the transverse sections of heavily drawn steels.

The diagonals of the imprint in the transverse section $\left(L r_{\mathrm{T}}\right.$ and $\left.L \theta_{\mathrm{T}}\right)$ diminish with the cumulative plastic strain but keep equal between them, whereas in the longitudinal section $\left(L r_{\mathrm{L}}\right.$ and $\left.L z_{\mathrm{L}}\right)$ they also diminish with the plastic strain, although enlarging the difference up to the $5 \%$ on the prestressing steel B7 (Fig. 4).

In the plastic strain appearing after the test, it is observed the existence of piling-up of the material surrounding the imprint boundary, which is itself related to the strain hardening coefficient $(n)$. Piling-up predominates in materials where $n<0.2$, it being higher as the exponent becomes lower. ${ }^{10)}$ The stress-strain curves obtained by the standard tension test under load in the longitudinal direction show in all cases hardening coefficients lower than 0.2. ${ }^{15}$ ) 
In the surfaces of the Vickers imprint the plastic strain through the contact perimeter is not uniform (Fig. 5): it is quite lower at the corners of the imprint due to the constrain imposed by the penetrator shape (the penetrator is not axisymmetric). In the transverse section the plastic strain is equal in the four sides of the imprint, while in the longitudinal one, and due to the increasing anisotropy of the steel as a consequence of cold drawing, the piling-up is higher in the vicinity of the diagonal perpendicular to the drawing direction with the associated increase of the difference between diagonals.

In order to observe the slip bands at a high scale, Rockwell C sharp indentation tests were performed, on the different steels, the results being shown in the Figs. 6 and 7. Initially the slip lines have an appearance of isotropic irregular mesh which evolves with cold drawing towards smaller sizes in the transverse section. In the longitudinal section the slip lines orientate in the axial direction during cold drawing, apart from the decrease of size.

Longitudinal cuts of Rockwell $\mathrm{C}$ imprints were made (Fig. 8), for both the hot rolled bar and the cold drawn wire by indenting over their transverse sections (where the imprint is circular) in sections as close as possible to those containing a diameter of the imprint and the loading axis. In the prestressing steel wire indented over its longitudinal section (where the imprint is elliptical) cuts were made in the

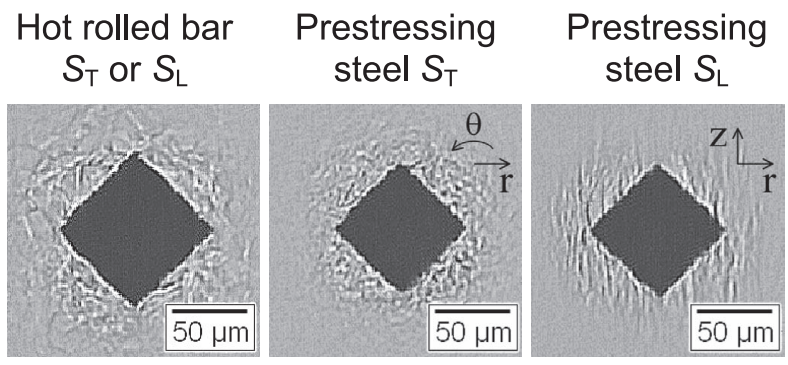

Fig. 5. Plastic deformation (Vickers imprint).

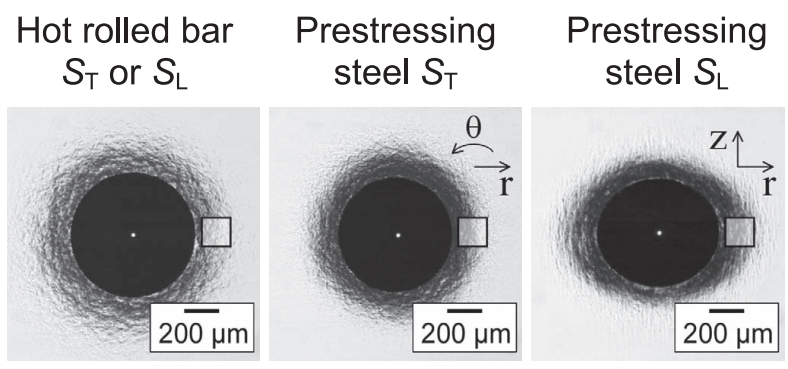

Fig. 6. Plastic deformation (Rockwell C imprint).

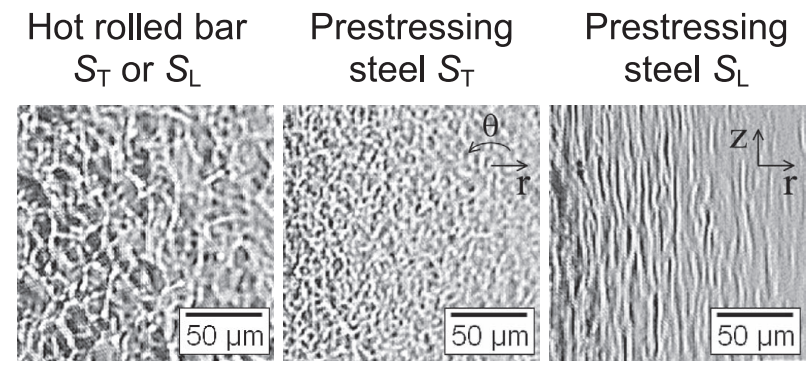

Fig. 7. Slip bands (detail of the Rockwell C imprint). sections containing the semiaxes of the ellipse and the loading axis. All of the afore-said cuts allow one to observe the distribution of piling-up surrounding the imprint, similar to that obtained in the Vickers tests, but using an axisymmetric indenter with conical shape.

\section{Numerical Modeling}

A three dimensional modelling of a Vickers microhardness test was performed by the finite element method (FEM) by means of the program Abaqus. ${ }^{16)}$ Only a quarter of the problem is necessary in steel B7 due to the symmetry (in B0 an eighth would be enough). The modelling consisted of introducing the penetrator in the material under load control and going back under displacement control (Fig. 9).

The indentator was characterised as a very stiff elastic material. The test sample has the minimum dimensions

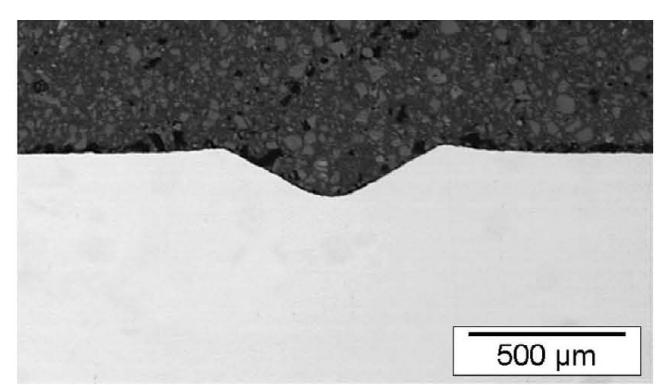

(a)

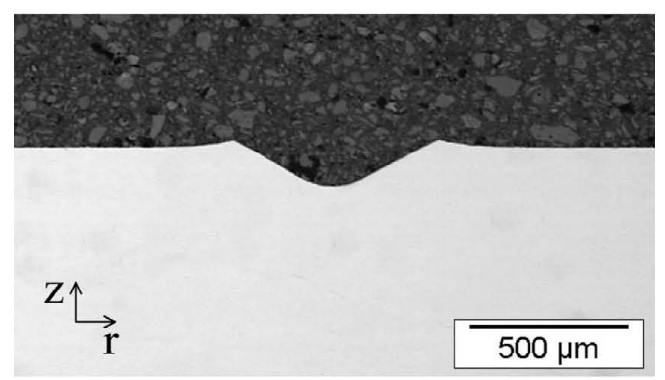

(b)
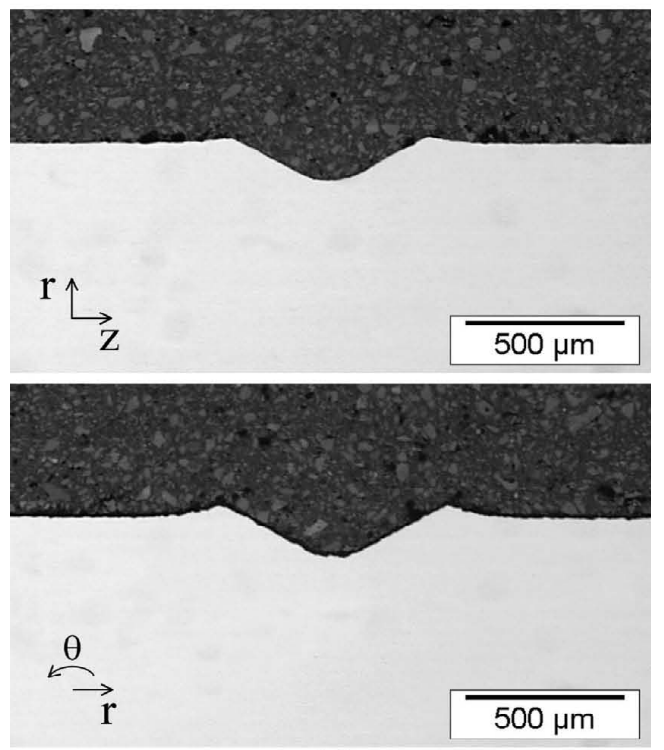

(c)

Fig. 8. Imprint profile: (a) B0, (b) B7, indentation in $S_{\mathrm{T}}$, (c) B7, indentation in $S_{\mathrm{L}}$. 

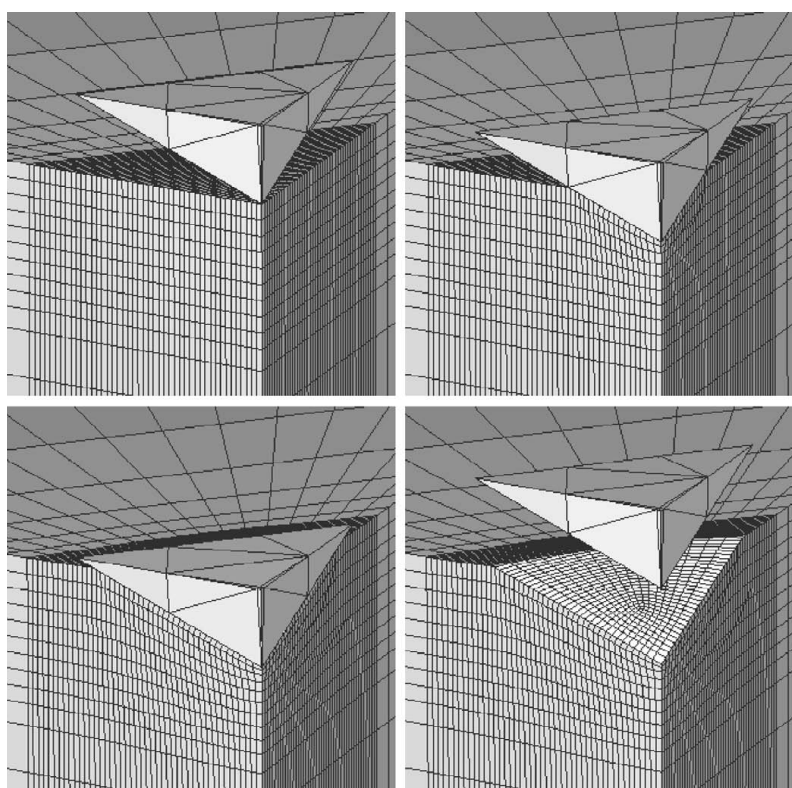

Fig. 9. Modelling of the Vickers hardness test (from left to right and from top to bottom).

required in the test. The finite elements were linear cubic elements of eight nodes (quadratic elements are not recommended for problems of contact simulations with Abaqus). Friction plays a relevant role in the response in the contact between solids exhibiting a great deal of piling-up, $\sim 0.15$ being an adequate value for the friction coefficient between diamond and the polished metallic surfaces. ${ }^{2,17)}$

The characteristic stress-strain $(\sigma-\mathcal{E})$ curves obtained by means of the tension test are known in the drawing direction, $\mathrm{cf.}^{15)}$ The anisotropic plastic behaviour of the material was determined by means of the Hill equation, ${ }^{18)}$ a criterion formulated in terms of a quadratic form on the basis of the components of the stress tensor:

$$
\begin{aligned}
& f\left(\sigma_{\mathrm{ij}}\right)=k_{11}\left(\sigma_{22}-\sigma_{33}\right)^{2}+k_{22}\left(\sigma_{33}-\sigma_{11}\right)^{2}+ \\
& k_{33}\left(\sigma_{11}-\sigma_{22}\right)^{2}+k_{23} \sigma_{23}^{2}+k_{31} \sigma_{31}^{2}+k_{12} \sigma_{12}^{2}-1
\end{aligned}
$$

The parameters defining the elastic domain for $f \leq 0$ are given by the three values of the tensile yield strength along the three orthotropy axes and the three values of the shear yield strength along the three symmetry planes. In the case of a prestressing steel wire analysed in this paper, transverse isotropy does exist in a plane perpendicular to the drawing axis, so that the number of parameters characterising the plastic model can be reduced as follows, cf.: ${ }^{11)}$

$$
\begin{aligned}
& \sigma_{11}^{0}=\sigma^{0} \\
& \sigma_{22}^{0}=\sigma_{33}^{0} \equiv \sigma_{t}^{0} \\
& \tau_{12}^{0}=\tau_{13}^{0} \equiv \tau^{0} \\
& \tau_{23}^{0}=\frac{\sigma_{t}^{0}}{\sqrt{3}}
\end{aligned}
$$

In the hot rolled bar, the diagonal of the imprint calculated by the FEM differs from the experimental value in less than $3 \%$. In the prestressing steel wire (where the material is not totally characterised due to the impossibility of exper-

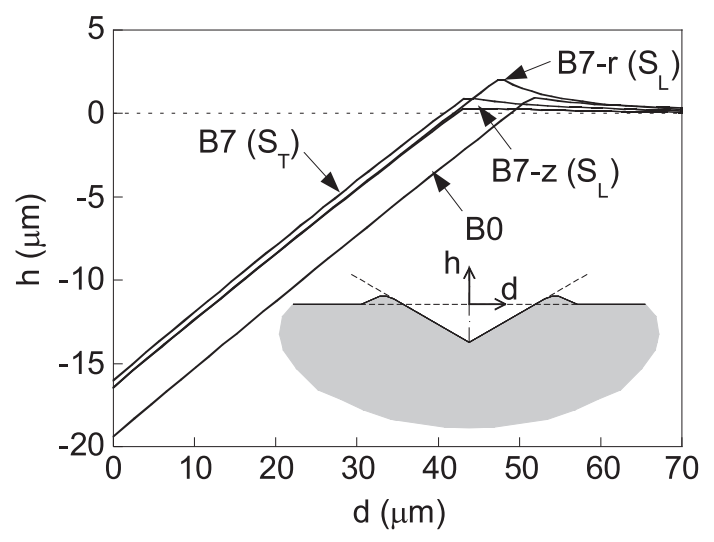

Fig. 10. Profile of the plastic deformation (FEM).

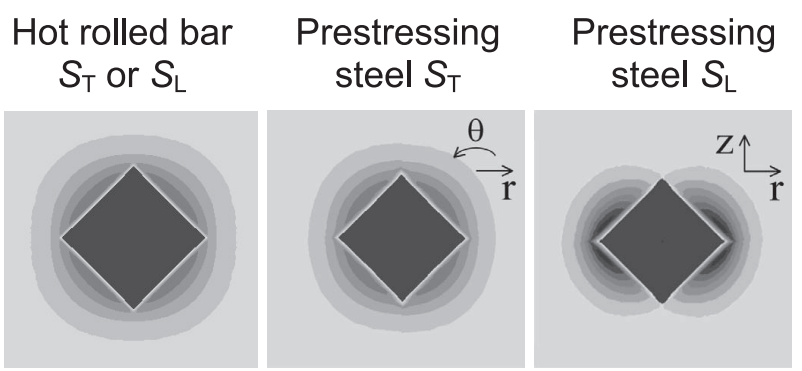

Fig. 11. Plastic deformation (numerical modelling of the Vickers indentation).

imental evaluation of the radial, or transversal, yield strength) approximate values of this yield strength parameter were fitted by using an error minimization function on the basis of the comparison between the experimental values of the imprint diagonals and the same diagonal lengths numerically predicted by the FEM, the latter computed in such a manner that different candidate values of the radial (or transversal) yield strength $\left(\sigma_{22}^{0}\right.$ or $\left.\sigma_{33}^{0}\right)$ were used in the computations, thus producing distinct imprints shapes (numerically predicted), so that the candidate value exhibiting the numerical imprint shape closer to the real one (experimentally obtained) was chosen. The obtained value was $20 \%$ lower than the corresponding to the longitudinal direction (axial or longitudinal yield strength $\sigma_{11}^{0}$ ), in a first approach considering $\tau^{0}=\sigma^{0} / \sqrt{3}$ and with a difference between imprint diagonals (experimental and numerical) lower than $5 \%$.

In cuts following planes perpendicular to the imprint and containing one of the diagonals of the latter, the imprint profile obtained by numerical simulation shows the variations in the piling-up shape as a consequence of the cold drawing process (Fig. 10). In the cutting plane where the imprint is analysed, the parameter $h$ indicates the imprint depth and $d$ the dimension containing the diagonal, the coordinate $(0,0)$ being the initial contact point of the indentator with the sample. It is seen that piling-up appears in both steels, it being isotropic (similar in all imprint diagonals) in the hot rolled bar and anisotropic (depending on the specific imprint diagonal) in the prestressing steel wire. In the latter case the piling-up effect is the highest in radial direction of the longitudinal section and the lowest in axial direction (drawing axis) of the same longitudinal section, and the piling-up being intermediate (between the two described limits) in the 


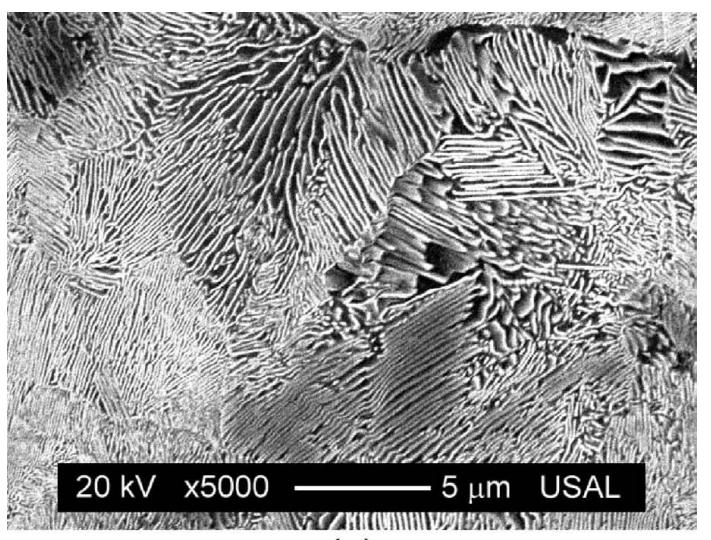

(a)

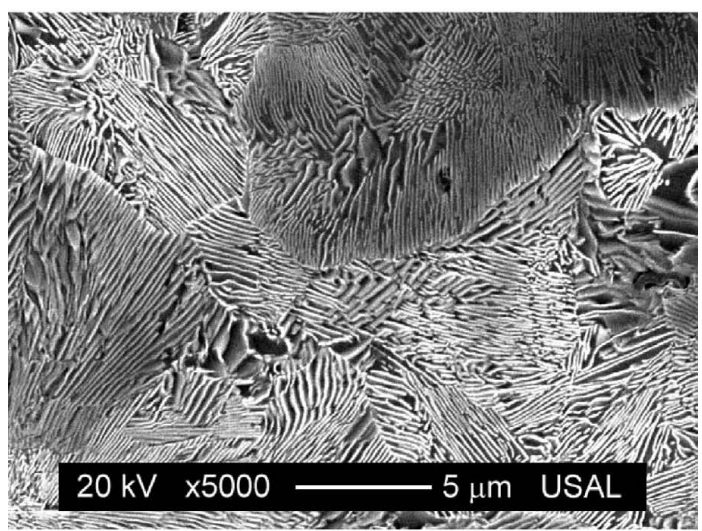

(b)

Fig. 12. Microstructure of steel B0: (a) transverse section, (b) longitudinal section.

case of the imprint located in the transverse section.

With regard to the plastic deformation surrounding the imprint, Fig. 11 shows that the numerically obtained appearance agrees with that experimentally evaluated (Fig. 5), i.e. material piling-up exhibits similar geometry.

\section{Discussion}

In Figs. 12 and 13 the microstructure is shown of both the hot rolled and the cold drawn steels in their characteristic sections, transverse and longitudinal, respectively. The horizontal side of the photograph is associated with the radial direction in the wire, whereas the vertical side of the photograph corresponds to the axial direction in the longitudinal section and to the annular direction in the transverse section.

The cold drawing process generates a marked microstructural orientation in the progressively drawn material which induces a clear anisotropy in the matter of plastic and fracture behaviour. In a transverse section of the cold drawn steel, a random orientation of both pearlitic colonies and lamellae is observed, together with an increasing packing closeness with cold drawing (i.e. the pearlite interlamellar spacing decreases with the drawing degree). On the other hand, in a longitudinal section of the same heavily drawn steel, the microstructural units evolve towards an oriented arrangement, becoming almost parallel to the wires axis or drawing direction. Such an orientation is associated with a longitudinal enlargement (with pearlite colonies slenderising) and a transverse shortening (and the afore-said increase

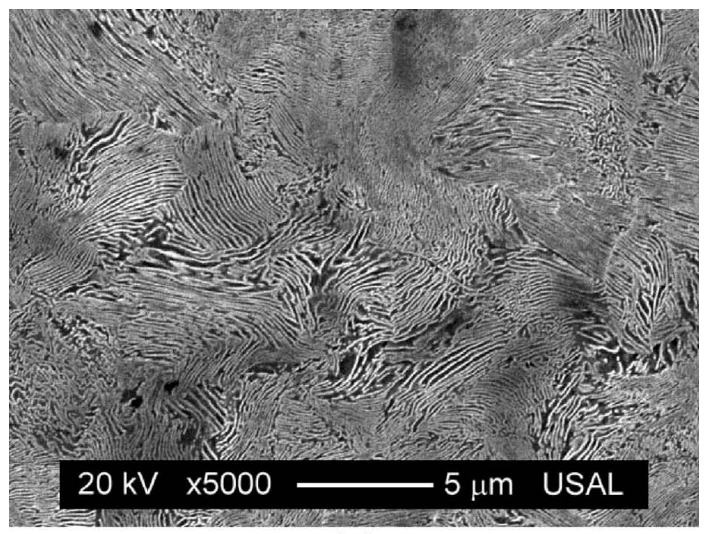

(a)

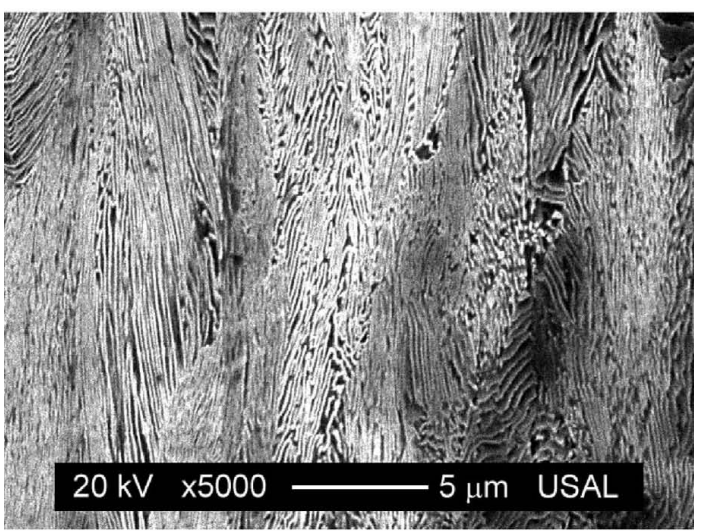

(b)

Fig. 13. Microstructure of steel B7: (a) transverse section, (b) longitudinal section.

of packing closeness and decrease of interlamellar spacing), cf. $^{19-22)}$

The hardness differences between the two analysed sections can be attributed to the microstructural variations during drawing, because the great number of interfaces (cementite layers) act as barriers to dislocation movement, and the number of interfaces (barriers) depends on the specific glide direction, thereby being a function of the directional hardening and therefore of the drawing degree. Thus in the cold drawn steel, plastic deformation exhibits directional dependence and shows privileged axes, so that the slip bands are parallel to the wire axis or drawing direction.

\section{Conclusions}

During cold drawing of eutectoid steel, an increase of dislocation density takes place, thus producing an increase of Vickers hardness of the prestressing steel in both longitudinal and transverse directions, together with radial variations associated with the presence of residual stresses in the material after cold drawing.

In progressively drawn steels, the hardness in the transverse section is higher than that in the longitudinal cut, the difference increasing with the degree of cold drawing. Such a phenomenon is related to the number of interfaces (cementite lamellae blocking dislocation movement), which modifies the shape and distribution of slip bands.

Numerical simulation by using a 3D finite element method allows a characterization of the anisotropic plastic behav- 
iour of the material on the basis of the imprint shape after indentation in all cases where a direct evaluation of plastic behaviour by means of a standard tension test is not possible due to the lack of material in the transverse direction.

Plastic anisotropy induced by the cold drawing process in pearlitic steel implies, for a plastic strain of 1.10, the existence of a radial or transversal yield strength, in a direction perpendicular to the drawing axis, of approximately $20 \%$ lower than the axial or longitudinal yield strength in the drawing direction.

\section{Acknowledgements}

The authors wish to acknowledge the financial support provided by the following Spanish Institutions: Ministry for Science and Technology (MCYT-FEDER; Grant MAT200201831), Ministry for Education and Science (MEC; Grant BIA2005-08965), Ministry for Science and Innovation (MCINN; Grant BIA2008-06810), Junta de Castilla y León (JCyL; Grants SA067A05, SA111A07 and SA039A08), and the steel supplied by EmeSA Trefilería (La Coruña, Spain).

\section{REFERENCES}

1) S. Jayaraman, G. T. Hahn, W. C. Oliver, C. A. Rubin and P. C. Bastian:
Int. J. Solids Struct., 35 (1998), 365.

2) D. Tabor: Hardness of Metals, Oxford University Press, Oxford, (1951), 192

3) P. L. Larsson: Int. J. Mech. Sci., 43 (2001), 895.

4) E. Giannakopoulos and S. Suresh: Scr. Mater., 40 (1999), 1191.

5) H. Pelletier: Tribol. Int., 39 (2006), 593.

6) Y. Murakami and M. Itokazu: Int. J. Solids Struct., 34 (1996), 4005.

7) O. Casals and J. Alcalá: Acta Mater., 53 (2005), 3545.

8) T. A. Venkatesh, K. J. Van Vliet, A. E. Giannakopoulos and S. Suresh: Scr. Mater., 42 (2000), 833.

9) J. L. Bucaille, S. Stauss, E. Felder and J. Michler: Acta Mater., 51 (2003), 1663.

10) J. Alcalá, A. C. Barone and M. Anglada: Acta Mater., 48 (2000), 3451.

11) M. Bocciarelli, G. Bolzon and G. Maier: Mech. Mater., 37 (2005), 855.

12) S. Carlsson and P. L. Larsson: Acta Mater., 49 (2001), 2179.

13) S. Suresh and A. E. Giannakopoulos: Acta Mater., 46 (1998), 5755.

14) J. M. Atienza, J. Ruiz-Hervias, M. L. Martínez-Pérez, F. J. Mompean, M. García-Hernández and M. Elices: Scr. Mater., 52 (2005), 1223.

15) F. J. Ayaso, B. González, J. C. Matos, D. Vergara, M. Lorenzo and J. Toribio: Anal. Mecán. Fract., 22 (2005), 128.

16) D. Hibbitt, B. Karlsson and P. Sorensen: Abaqus User's Manual v6.3, Abaqus Inc., Providence, (2003)

17) M. Mata and J. Alcalá: J. Mech. Phys. Solids, 52 (2004), 145.

18) R. Hill: The Mathematical Theory of Plasticity, Clarendon Press, Oxford, (1998), 366.

19) J. Toribio and E. Ovejero: Mater. Sci. Eng. A, 234-236 (1997), 579.

20) J. Toribio and E. Ovejero: J. Mater. Sci. Lett., 17 (1998), 1045.

21) J. Toribio and E. Ovejero: Scr. Mater., 39 (1998), 323.

22) J. Toribio and E. Ovejero: Mech. Time-Depend. Mater., 1 (1998), 307. 